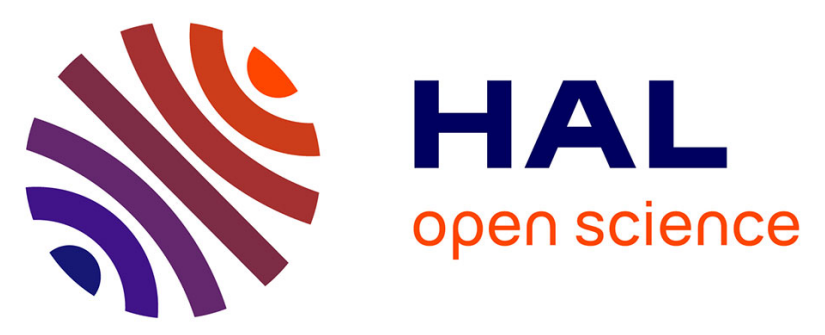

\title{
Application des recommandations dans la prise en charge du cancer de l'endomètre en pratique clinique. Étude rétrospective bretonne
}

O. de Kerdaniel, N. Body, E. Davoine, F. Foucher, Sébastien Henno, A.

Tavenard, B. Laviolle, C. Rozel, M. Leblanc, V. Lavoue, et al.

\section{To cite this version:}

O. de Kerdaniel, N. Body, E. Davoine, F. Foucher, Sébastien Henno, et al.. Application des recommandations dans la prise en charge du cancer de l'endomètre en pratique clinique. Étude rétrospective bretonne. Journal de Gynécologie Obstétrique et Biologie de la Reproduction, 2016, 45 (9), pp.10451053. 10.1016/j.jgyn.2015.12.009 . hal-01259220

\section{HAL Id: hal-01259220}

https://hal-univ-rennes1.archives-ouvertes.fr/hal-01259220

Submitted on 28 Jan 2016

HAL is a multi-disciplinary open access archive for the deposit and dissemination of scientific research documents, whether they are published or not. The documents may come from teaching and research institutions in France or abroad, or from public or private research centers.
L'archive ouverte pluridisciplinaire HAL, est destinée au dépôt et à la diffusion de documents scientifiques de niveau recherche, publiés ou non, émanant des établissements d'enseignement et de recherche français ou étrangers, des laboratoires publics ou privés. 


\section{How were used recommendations for endometrial carcinoma? Britain retrospective} study.

De Kerdaniel O, ${ }^{1}$ Body N, ${ }^{1}$ Davoine E, ${ }^{1}$ Foucher F, ${ }^{1,5}$ Henno S, ${ }^{2}$ Tavenard A, ${ }^{3}$ Laviolle B, ${ }^{3}$ Rozel $\mathrm{C}^{4}$, Leblanc $\mathrm{M}^{7}$ Lavoue $\mathrm{V},{ }^{1,5,6^{*}}$, Levêque J.,5,6

${ }^{1}$ CHU de Rennes, Service de gynécologie, Hôpital Sud, Rennes. France

${ }^{2} \mathrm{CHU}$ de Rennes, service d'Anatomopathologie, CHU Pontchaillou, Rennes. France

${ }^{3}$ CHU de Rennes, service de Pharmacologie Clinique, CIC Inserm 0203, Hôpital Pontchaillou, Rennes. France

${ }^{4}$ CHU de Rennes, Service de Radiologie, Hôpital Sud, Rennes. France

${ }^{5}$ Oncogenesis, Stress and Signaling, ER 4440, CRCL CRLCC Eugène Marquis, Rennes. France

${ }^{6}$ Université de Rennes 1, Faculté de médecine, Rennes, France.

${ }^{7}$ Centre Hospitalier Bretagne Atlantique, Service de Gynécologie, Vannes. France

\section{Corresponding authors:}

*Vincent Lavoué, CHU de Rennes, Service de gynécologie, Hôpital Sud, Rennes, France. Oncogenesis, Stress and Signaling, ER 4440, CRCL CRLCC Eugène Marquis, Rennes. France. Université de Rennes 1, Faculté de médecine, Rennes, France. Vincent.lavoue@churennes.fr

Conflit d'intérêt : Les auteurs ne declarent aucun conflit d'intérêt.

Titre courant : Applications des recommandations dans la prise en charge du cancer de l'endomètre. 


\section{Résumé}

Objectif : Evaluer l'application des recommandations de l'Institut National du Cancer concernant la prise en charge diagnostique et thérapeutique du cancer de l'endomètre.

Méthodes : Etude rétrospective observationnelle portant sur 137 patientes traitées pour un cancer de l'endomètre entre 2011 et 2013.

Résultats : Le bilan préopératoire était conforme aux recommandations dans $66,4 \%$ des cas et sous-estimait le risque d'envahissement ganglionnaire dans $44,9 \%$ des cas. La sous-estimation du risque sur le bilan préopératoire entraînait une sous-stadification chirurgicale dans $26,3 \%$ des cas.

Conclusion : Pour améliorer la pertinence du bilan pré-opératoire dans le cancer de l'endomètre, une expertise radiologique et anatomo-pathologique est nécessaire sur le même modèle que ce qui est réclamé pour la prise en charge chirurgicale. Cependant, même réalisée de manière optimale, l'évaluation pré-opératoire peine à proposer une stratification du risque d'envahissement ganglionnaire satisfaisante dans le cancer de l'endomètre poussant à modifier les prises en charges chirurgicales recommandées en associant par exemple le prélèvement $\mathrm{du}$ ganglion sentinelle en association à l'hystérectomie dans les cancers de l'endomètre estimés à bas risque.

Mots clés : Cancer de l'endomètre ; IRM ; anatomo-pathologie ; stratification du risque 


\begin{abstract}
:
Objective: To assess the use of French Cancer Institute recommendations for the diagnosis and treatment of endometrial cancer.

Methods: Retrospective observational study involving 137 patients with endometrial cancer between 2011 and 2013.

Results: Both MRI and pathological assessment with correct report as recommended were used for $66.4 \%$ of patients with endometrial cancer. For patients with correct pre-operative assessment, $44.9 \%$ of patients were uncorrectly classified and upgraded on final pathological analysis of hysterectomy concerning lymph node involvement risk. These patients did not have confident surgical assessment, according this risk.

Conclusion: To improve relevance of preoperative assessment in endometrial cancer, radiological and pathological expertise is required. However, even performed optimally, preoperative assessment does not allow correct risk stratification of lymph node involvement in endometrial cancer. This ineffective stratification leads to propose sentinel lymph node biopsy with hysterectomy in case of pre-operative low risk endometrial cancer assessment.
\end{abstract}

Key words: endometrial cancer; MRI; pathological analysis; risk classification 


\section{$\underline{\text { Introduction }}$}

Le cancer de l'endomètre (CE) est le cancer gynécologique le plus fréquent en France, se situant au $4^{\text {ème }}$ rang des cancers de la femme avec 7275 nouveaux cas estimés en $2012^{(1)}$. Son incidence n'a cessé de croitre ces dernières années en raison de l'augmentation de l'espérance de vie et de la prévalence de l'obésité. Les patientes sont habituellement diagnostiquées à un stade précoce, lorsque la tumeur est limitée au corps de l'utérus avec une survie globale allant de $85 \%$ à $91 \%{ }^{(2,3)}$.

La publication par l'Institut National du Cancer (INCa) en 2010 de nouvelles recommandations sur la prise en charge du cancer de l'endomètre a changé les pratiques chirurgicales en tendant vers une désescalade thérapeutique, notamment pour les cancers estimés à risque faible de récidive et d'envahissement ganglionnaire ${ }^{(4)}$. Le bilan préopératoire comprend un prélèvement endométrial et une Imagerie par Résonance Magnétique (IRM) pelvienne et lomboaortique permettant d'évaluer le stade tumoral, le type et le grade histologique. Ce bilan permet de classer la patiente en terme de risque de récidive et de risque de métastases ganglionnaires et de guider le geste chirurgical en posant l'indication de lymphadénectomies associées à l'hystérectomie ${ }^{(2,4-11)}$.

Cette étude est une enquête de pratique évaluant le respect des recommandations de l'INCa sur la prise en charge diagnostique et thérapeutique du cancer de l'endomètre quatre ans après leur diffusion.

\section{Matériel et méthodes}

\section{Patientes et design de l'étude}

Il s'agit d'une étude rétrospective observationnelle portant sur les patientes traitées pour un cancer de l'endomètre entre le $1^{\text {er }}$ janvier 2011 et le 31 décembre 2013 au sein du Centre Hospitalier Universitaire (CHU) de Rennes et du Centre Hospitalier Régional (CHR) de Vannes. 
Le critère d'inclusion était la présence d'un cancer de l'endomètre quelque soit son stade, son type et son grade. Les critères d'exclusion étaient l'absence de chirurgie réalisée et le diagnostic fortuit de CE sur pièce d'hystérectomie.

Les données étaient recueillies à partir des dossiers cliniques des deux établissements cités et portaient sur les caractéristiques de la patiente (âge au moment du diagnostic de cancer de l'endomètre, obésité (Indice de Masse Corporelle (IMC) $\geq 30 \mathrm{~kg} / \mathrm{m} 2$ ), statut ménopausique, prise d'un traitement hormonal de la ménopause), le bilan préthérapeutique anatomopathologique (type de prélèvement, type histologique, grade histo-pronostique), le bilan préthérapeutique radiologique (type d'examens d'imagerie : échographie, Imagerie par résonnance Magnétique (IRM), Tomodensitométrie (TDM)) avec l'estimation de l'infiltration myométriale et la recherche d'adénopathies, le stade du cancer selon la Fédération Internationale de Gynécologie Obstétrique $(\mathrm{FIGO})^{(5)}$, la classification pré et postopératoire du risque de récidive et/ou d'envahissement ganglionnaire selon la classification de l'European Society for Medical Oncology (ESMO) $)^{(6-9)}$ et de l' $\mathrm{INCa}^{(4)}$, le type de chirurgie réalisée, la voie d'abord, la concordance radiohistologique pré et postopératoire, l'existence d'une reprise chirurgicale et les traitements adjuvants réalisés.

Le critère principal de jugement de cette étude était l'écart par rapport aux recommandations de l'INCa.

Cet écart était défini sur :

- le bilan préthérapeutique par sa non réalisation ou par l'absence d'éléments attendus sur le compte rendu d'IRM ${ }^{(12)}$ (par exemple l'absence d'envahissement myométrial non précisé) ou par l'absence de description d'éléments attendus sur l'histologie (absence de précision sur le grade par exemple) nécessaires à la classification préopératoire du risque d'envahissement ganglionnaire

- la prise en charge chirurgicale (surstadification chirurgicale par la réalisation d'une lymphadénectomie dans une classification à bas risque en préopératoire ou post-opératoire ou sous-stadification par l'absence de lymphadénectomie recommandée dans une classification à haut risque en pré- ou post-opératoire) 
- et les traitements adjuvants (sur-traitement par la réalisation d'une radiothérapie externe ou d'une curiethérapie non recommandées ou sous-traitement par l'absence de réalisation d'une radiothérapie externe ou d'une curiethérapie recommandées).

L'objectif principal de l'étude était d'évaluer l'écart de la prise en charge du CE par rapport aux recommandations.

Les objectifs secondaires étaient d'évaluer la corrélation entre cet écart et les comorbidités des patientes notamment en ce qui concerne la stadification chirurgicale et de rechercher un lien entre un excès d'irradiation et une sous stadification chirurgicale.

\section{Bilan préthérapeutique}

Le prélèvement endométrial était réalisé à l'aide d'une pipelle de Cornier $^{\circledR}$ ou par hystéroscopie -curetage. L'analyse anatomopathologique permettait de distinguer 2 types histologiques: le type 1 représentant les tumeurs endométrioïdes (et les tumeurs mixtes associant une composante mucineuse ou villoglandulaire) classées en 3 grades selon le pourcentage de contingent indifférencié et le type 2 regroupant les carcinomes à cellules claires, les carcinomes papillaires/séreux et les carcinosarcomes.

Le bilan d'extension locorégionale recommandé reposait sur l'IRM pelvienne associée à une exploration IRM des aires ganglionnaires lomboaortiques ${ }^{(12)}$.

D’après la classification de mai 2009 de la $\mathrm{FIGO}^{(5)}$, l'infiltration myométriale était classée en stade IA/T1a pour les tumeurs limitées à l'endomètre ou ne dépassant pas la moitié du myomètre et en stade IB/T1b pour celles envahissant la moitié ou plus de la moitié du myomètre. La suspicion d'adénopathie était définie au niveau radiologique par un axe ganglionnaire des ganglions locorégionaux de plus de $10 \mathrm{~mm}$. 
Selon la classification de l'European Society for Medical Oncology (ESMO), un risque bas (RB) de récidive ou d'envahissement ganglionnaire était défini par les stades IA/T1a, de grade 1 ou 2, de type 1 histologique, un risque intermédiaire (RI) par les stades IA/T1a, de grade 3, de type 1 histologique et par les stades IB/T1b, de grade 1ou 2, de type 1 histologique et un risque élevé (RE) par les stades IB/T1b, de grade 3, de type 1 histologique. ${ }^{(6-9)}$ En cas d'adénopathie à l'IRM, la patiente a été classée dans le groupe RE.

L'INCa a modifié cette classification du risque en prenant en compte la présence d'emboles lymphovasculaires faisant classer la tumeur automatiquement en RE lorsque celles-ci étaient présentes.

Par simplification, nous avons considéré les tumeurs de type 2 histologique et les tumeurs de type 1 histologique ayant un stade FIGO $\geq 2$ comme étant RE.

\section{Chirurgie et traitements adjuvants}

Les recommandations de l'INCa de $2010^{(4)}$ préconisent pour les patientes présentant un CE à risque bas une hystérectomie totale non conservatrice (HTNC) suivie d'une simple surveillance. En cas de cancer à risque intermédiaire de récidive, l'HTNC peut être associée à un curage pelvien $(\mathrm{CP})$ suivie d'une curiethérapie à haut débit de dose. Les patientes présentant un cancer à risque élevé doivent bénéficier d'une HTNC associé à un curage lombo-aortique plus ou moins associé à un $\mathrm{CP}$ suivi d'une radiothérapie externe et d'une curiethérapie. A noter, les patientes avec un cancer de type 2 histologique de type carcinome à cellules claires ou papillaire/séreux doivent bénéficier systématiquement d'un $\mathrm{CP}$ associé à un CLA et d'une omentectomie infracolique.

En cas de cancer de stade II de la FIGO, une chirurgie comprenant une HTNC simple ou élargie (afin d'obtenir des marges saines) associée à un CP plus ou moins à un CLA doit être réalisée et en cas d'envahissement local important, les patientes peuvent bénéficier d'une irradiation préopératoire. 
Les patientes avec un cancer de stade IIIA doivent bénéficier d'une HTNC avec CP, CLA et omentectomie infra-gastrique suivi d'une irradiation. La chimiothérapie est discutée au cas par cas dans les types 2 histologique, les atteintes annexielles et les stades FIGO $\geq$ IIIb.

Les patientes avec un cancer de stade IIIB doivent bénéficier d'une irradiation première (un CLA préthérapeutique peut être discuté), une chirurgie pouvant être envisagée en cas de réponse incomplète au décours de la radiothérapie. Les patientes présentant un $\mathrm{CE}$ de stade IIIC ont une HTNC avec CP et CLA suivie d'une irradiation. Les patientes avec un cancer de stade IVA doivent bénéficier d'une irradiation et plus ou moins d'une exentération pelvienne en cas d'échec de l'irradiation et celles avec un cancer de stade IVB d'une chirurgie de cytoréduction complète à visée curative (type ovaire) en cas de carcinose résécable et de l'absence de métastase.

\section{Analyse statistique}

Le test du $\mathrm{Chi}^{2}$ ou le test exact de Fisher ont été utilisés pour évaluer la relation entre un traitement chirurgical ou adjuvant non recommandé et les comorbidités (âge et obésité) et pour la relation entre un excès de traitement adjuvant et une sous stadification chirurgicale.

Une valeur de $\mathrm{p}<0,05$ a été retenue comme statistiquement significative. Les données étaient gérées par une base de données Excel (Microsoft, Redmond, WA, USA) et analysées à l'aide du logiciel statistique R (www.r-project.org). 


\section{$\underline{\text { Résultats }}$}

\section{Caractéristiques de la population}

De janvier 2011 à décembre 2013, 150 patientes ont été prises en charge pour un cancer de l'endomètre (96 au CHU de Rennes et 54 au CHR de Vannes). Treize patientes ont été exclues de cette étude ( 6 patientes non opérées, 7 diagnostics de $\mathrm{CE}$ fortuits sur pièce opératoire d'hystérectomie). Au final, 137 patientes ont été incluses dans notre étude. La moyenne d'âge des patientes était de $68 \pm 10$ ans. L'indice de masse corporelle moyen était de $30,7 \pm 7,8 \mathrm{~kg} / \mathrm{m}^{2}$. Les caractéristiques des patientes sont reportées dans le tableau 1 .

\section{Bilan préopératoire}

Les biopsies avaient été réalisées par pipelle de Cornier $^{\circledR}$ et hystéroscopie-curetage dans $51,1 \%(n=70 / 317)$ et $47,4 \%$ des cas $(n=65 / 137)$ respectivement.

Le tableau 2 résume les caractéristiques anatomopathologiques et radiologiques du bilan préopératoire.

Le type histologique était caractérisé dans $95,6 \%(n=131 / 137)$ des cas et le grade pour les tumeurs de type I histologique précisé dans 92,1\%(n=105/114) des cas.

Quatre vingt trois $\mathrm{p}$. cent $(\mathrm{n}=114 / 137)$ des prélèvements retrouvaient une tumeur de type I histologique, 12,4\% (n=17/137) une tumeur de type II et 4,4\% (n=6/137) étaient non renseignés.

Les tumeurs de type I histologique étaient réparties en grade 1, 2,3 et indéterminé dans 43,8\% $(n=50), 43 \%(n=49), 5,3 \%(n=6)$ et $7,9 \%(n=9)$ des cas respectivement.

L'IRM était réalisée dans 74,5\% (n=102/137) des cas. En cas d'IRM, l'exploration pelvienne et lomboaortique des aires ganglionnaires et l'exploration de la profondeur de l'envahissement myométrial n'était effective que dans 66,4\% (n=91/137).

Un envahissement supérieur ou égal à 50\% de la profondeur du myomètre était retrouvé dans 29,9\% (n=41/137) des cas et un envahissement ganglionnaire était suspecté dans $12,4 \%$ $(n=17 / 137)$ des cas. 
En ce qui concerne la classification du risque, parmi le sous-groupe de patientes avec un type 1 histologique, on retrouvait 45 patientes $(65,2 \%)$ à risque bas, $24(34,8 \%)$ à risque intermédiaire et aucune à risque élevé.

Compte tenu de l'ensemble des données du bilan préopératoire, seul 74,5\% (n=102/137) des patientes ont pu bénéficier d'une classification du risque d'envahissement ganglionnaire selon les recommandations de l'INCa.

Concernant l'un des objectifs principaux de cette étude, il est montré que seules 91 patientes $(66,4 \%)$ avaient un bilan préopératoire complet avec un compte rendu d'imagerie et un compte rendu anatomo-pathologique satisfaisants tels que recommandés par l'INCa pour permettre une classification correcte des patientes en terme de risque d'envahissement ganglionnaire.

\section{Chirurgie}

La voie d'abord chirurgicale était coelioscopique dans 71,5\% (n=98) des cas. Une laparotomie ou une voie vaginale avaient été réalisées dans $24,8 \%(n=34)$ et $3,7 \%$ des cas $(n=5)$ respectivement. L'exploration ganglionnaire avait été réalisée par une technique du ganglion sentinelle, un curage pelvien ou un curage lomboaortique dans $8 \%(n=11), 55,4 \%$ $(\mathrm{n}=76)$ et $14,5 \%$ des cas $(\mathrm{n}=20)$ respectivement.

La prise en charge chirurgicale en fonction de la classification du risque pré et postopératoire est détaillée dans le tableau 3.

A partir du risque défini selon le bilan préopératoire, une stadification chirurgicale recommandée était réalisée dans $51,1 \%(\mathrm{n}=70)$ des cas, 14,6\% (20/137) des patientes ayant été sur-stadées et 8,8\% (12/137) sous-stadées.

Trente-cinq patientes $(25,5 \%)$ ont été opérées sans classification correcte préopératoire du risque et parmi elles, dix-sept ont eu une évaluation ganglionnaire chirurgicale. Parmi ces 35 patientes, selon la classification du risque postopératoire, $14(40 \%)$ ont été sous-stadées chirurgicalement et $8(23 \%)$ sur-stadées chirurgicalement. 
$\mathrm{Au}$ final, à partir du risque défini selon l'histologie postopératoire, la prise en charge chirurgicale était en accord avec les recommandations dans 73,7\%(101/137) des cas et 26,3\% des patientes ont eu une sous-stadification chirurgicale.

Il n'a pas été mis en évidence de corrélation statistiquement significative entre l'obésité et la sous-stadification chirurgicale que ce soit en fonction du risque défini par le bilan préopératoire ou par l'histologie définitive (par exemple sur le risque défini en postopératoire, $31,2 \%(20 / 64)$ des patientes avec un IMC $\geq 30$ sont sous-stadées versus 20,5\%( $15 / 73)$ des patientes avec un IMC $<30(\mathrm{NS}))$.

En ce qui concerne l'âge, 33,9\%(19/56) des patientes de plus de 70 ans sont sous-stadées selon le risque défini en postopératoire contre $19,7 \%(16 / 81)$ des patientes de moins de 70 ans $(p=0,0370)$.

Par ailleurs, sur les 12 patientes sous-stadées lors d'une chirurgie initiale (8,8\%), 4 ont été réopérées pour être conforme à la stadification chirurgicale tel que recommandé par l'INCa (2 cas de stade FIGO sous estimé en préopératoire et 2 cas de type 2 histologique postopératoire qui étaient diagnostiqués de type 1 en préopératoire).

\section{Anatomopathologie postopératoire}

Les caractéristiques histologiques postopératoires sont résumées dans le tableau 4.

Quatre-vingt six p. cent (118/137) des patientes présentaient un type 1 histologique (endométrioïde) et 14\% (19/137) un type 2 histologique.

En ce qui concerne le stade FIGO définitif, la population étudiée regroupait 70,8\% de stade FIGO IA ou IB et $29,2 \%$ de stades II et supérieurs.

Seize patientes sur $137(11,6 \%)$ présentaient en postopératoire un envahissement ganglionnaire dont 9 avec une histologie de type 1 et 7 une histologie de type 2 .

Des emboles lymphovasculaires étaient retrouvés dans 35,7\% $(n=49)$ des cas et il s'agissait dans $46,9 \%$ des cas de cancer de stade I et dans $69,4 \%$ des cas de cancer de type 1 histologique.

Parmi les 16 patientes $\mathrm{N}+$ en postopératoire, des emboles étaient détectés chez $14(87,5 \%)$ d'entre elles. 
En comparant les groupes de risques définis par le bilan préopératoire et par l'histologie définitive, on observait que :

- sur les 45 patientes estimées à $\mathrm{RB}$ en préopératoire, $40 \%$ ont été classées à risque supérieur (RB ou RE) à l'histologie définitive.

- sur les 24 patientes estimées à RI en préopératoire, 54\% ont été classées en RE à l'histologie définitive.

$\mathrm{Au}$ total, $44,9 \%$ des patientes estimées à $\mathrm{RB}$ ou RI en préopératoire ont été surclassées en terme de risque sur l'histologie définitive.

Il est à noter que sur les 24 patientes classées à RE sur l'histologie postopératoire mais classées à $\mathrm{RB}$ ou RI en fonction du bilan préopératoire, $12(50 \%)$ l'ont été du fait de la présence d'emboles sur la pièce opératoire et aucune n'a bénéficié d'une reprise de stadification ganglionnaire.

\section{Traitement adjuvant}

Quarante et une patientes $(30,1 \%)$ n'ont pas bénéficié de traitements adjuvants.

Quatre-vingt treize patientes $(68,3 \%)$ ont bénéficié d'une irradiation complémentaire (29 curiethérapies seules, 16 radiothérapies seules, 48 curiethérapies et radiothérapies associées). Dix sept patientes (12,5\%) ont bénéficié d'une chimiothérapie adjuvante.

Parmi les 137 patientes, 86\% avaient eu un traitement adjuvant en accord avec les recommandations et basé sur le niveau de risque ( 18 patientes en désaccord avec les recommandations dont 5 ayant eu une irradiation par « excès » et 13 n'ayant pas bénéficié d'une irradiation pourtant recommandée).

Parmi les 5 patientes ayant été sur-traitées, une patiente avait une stadification chirurgicale non conforme (pas de lymphadénectomie).

Il n'y a pas de lien significatif entre le sous ou sur-traitement adjuvant et l'âge et /ou l'obésité des patientes.

Parmi les 66 patientes à risque élevé, 61 (92,4\%) ont eu une irradiation.

Treize patientes sur 15 classées à risque élevé sur la seule présence des emboles lymphovasculaires ont bénéficié d'une irradiation. 


\section{$\underline{\text { Discussion }}$}

Notre étude a permis de montrer que le bilan préopératoire, comprenant IRM et biopsie endométriale, n'était conforme aux recommandations que dans seulement $61,3 \%$ des cas. Ce bilan préopératoire sous-estimait le risque d'envahissement ganglionnaire tel que défini par l'INCa chez $44,9 \%$ des patientes aboutissant à une sous-stadification chirurgicale dans 26,3 $\%$ des cas. Cette sous-estimation du risque ne semblait pas entrainer la réalisation de traitements adjuvants en « excès ».

Les données préopératoires fournies par la biopsie d'endomètre et par l'IRM pelvienne et lombo-aortique sont essentielles pour pouvoir distinguer les patientes à risque faible de récidive des patientes à risque élevé, chez qui l'exploration ganglionnaire permettra de déterminer le pronostic et guidera les décisions quant à la nécessité de mettre en œuvre des traitements adjuvants ${ }^{(2,4,6-11,13-15)}$. Dans un quart des cas, l'IRM, qui malgré des performances hétérogènes selon les études, reste le meilleur examen pour évaluer l'envahissement myométrial, cervical et ganglionnaire, ${ }^{(16-18)}$ n'était pas réalisée.

S'agissant d'une enquête d'évaluation des pratiques, notre étude souffre d'un certain nombre de faiblesse du fait de son caractère rétrospectif notamment l'existence d'un biais dû à la perte d'information. Cependant nos résultats concordent avec la littérature, et ils reflètent en pratique la réalité de prise en charge du cancer de l'endomètre. En effet, nos résultats sont similaires à l'étude multicentrique de Raimond et al. publiée en 2014 qui indique que 26,7\% ( 43/161) des patientes n'avaient pas bénéficié d'une IRM ${ }^{(15)}$. Le délai moyen d'obtention d'un rendez-vous pour une IRM en Bretagne est de 41,5 jours, pouvant expliquer ce taux de non -réalisation dans un contexte de cancer, revêtant souvent un certain caractère d'urgence au moins psychologique pour les patientes et parfois pour le praticien. Notre étude indique également que l'on sous estimait le risque d'envahissement ganglionnaire dans $48,9 \%$ des cas avec théoriquement un geste chirurgical non conforme pour $48,9 \%$ des patientes. 
Cependant le non respect strict des recommandations sur le geste chirurgical avec la réalisation de lymphadénectomies non recommandées au vu du bilan pré-opératoire permet de diminuer ce taux de gestes chirurgicaux non conformes à 26,3 si l'on tient compte du risque défini par l'histologie définitive, concordant avec la série de Raimond et al (27,3\%).

Cette sous estimation du risque était due à la fois à la discordance anatomoradiologique pré et postopératoire et à la présence d'emboles lymphovasculaires découverts sur la pièce opératoire d'hystérectomie, ce qui concordait avec la littérature ${ }^{(15,16-18,19-21)}$. En effet, les emboles représentent un facteur prédictif puissant d'envahissement ganglionnaire ${ }^{(22-24)}$ et impactent la survie globale et sans récidive ${ }^{(25,26)}$. Aristizabal et al considèrent leur présence comme plus pertinente que la distinction entre les stades IA et IB en terme de prédiction de survie $^{(27)}$. En ajoutant les emboles dans son modèle de prédiction du risque, l'INCa a modifié la stratégie opératoire sans prendre en compte le fait qu'on ne peut pas les identifier en préopératoire $^{(28)}$.

La logique des recommandations voudrait que l'on réopère les patientes sous stadées chirurgicalement initialement. Ce qui n'est pas fait en pratique devant la morbidité d'une réintervention chez ces patientes souvent âgées et/ou obèses ${ }^{(29,30)}$. En effet, on a observé une corrélation significative entre les patientes sous stadées chirurgicalement et l'âge supérieur à 70 ans.

Les traitements adjuvants administrés dans notre étude respectaient les recommandations dans la plupart des cas, à savoir la surveillance dans les cancers à risque bas de récidive, une irradiation dans les cancers à risque intermédiaire et élevé et l'administration de chimiothérapie dans les cancers de stade avancé ${ }^{(31)}$.

La désescalade thérapeutique initiée par l'ESMO et par l'INCa est fondée sur deux études prospectives randomisées qui montraient une absence de bénéfice à réaliser un curage ganglionnaire en terme de survie globale et de survie sans récidive dans les groupes à faible risque de récidive ${ }^{(32,33)}$. 
La méta-analyse de Frost et al confirme ces données ${ }^{(34)}$. Cependant, l'essai prospectif SENTIENDO montre que $11 \%$ des patientes à risque bas et $15 \%$ des patientes à risque intermédiaire présentaient un ganglion sentinelle métastatique et parmi elles, $47 \%$ avaient des micrométastases diagnostiquées uniquement en immunohistochimie ${ }^{(35)}$. La question actuelle est de pouvoir proposer une évaluation ganglionnaire chirurgicale au prix d'une morbidité faible. Pour améliorer la prise en charge des cancers de l'endomètre, deux options seraient envisageables : l'amélioration du bilan préopératoire en augmentant la sensibilité de détection des emboles (ce qui n'est pas possible actuellement) ou l'évaluation ganglionnaire par la technique du ganglion sentinelle chez les patientes à bas risque. Les recommandations actuelles de prises en charge du cancer de l'endomètre prennent surtout le risque de sousestimation de la maladie. La difficulté est double : i) le bilan pré-opératoire basé sur l'IRM et l'anatomo-pathologie n'est pas suffisamment concordant avec l'histologie définitive avec le plus souvent une sous-estimation de la maladie cancéreuse et ii) même si la maladie est correctement classée dans le groupe à bas risque ou à risque intermédiaire, il existe encore un risque de $10 \%$ à $15 \%$ de métastases ganglionnaires occultes. Ces patientes estimées à bas risques ne reçoivent pas de radiothérapie pelvienne complémentaire et donc voient leur risque de récidive ganglionnaire augmenter significativement. Ces patientes sont potentiellement curables lors de leur maladie initiale car la radiothérapie initiale permet de stériliser habituellement une maladie micrométastatique ganglionnaire.

\section{Conclusion}

Il est nécessaire d'améliorer la stadification préopératoire par la réalisation systématique d'une histologie et d'une IRM préopératoire. Le bilan préopératoire actuellement recommandé sous estime le risque d'envahissement ganglionnaire et de récidive pour environ la moitié des patientes. Cette sous-estimation est due en partie à l'introduction des emboles lymphovasculaires dans la stratification du modèle de risque, non détectables en préopératoire. 
La désescalade thérapeutique pour les cancers de l'endomètre à risque bas ou intermédiaire d'envahissement ganglionnaire évalué sur le bilan pré-opératoire expose donc les patientes à une morbidité plus élevée en cas de réintervention ou à un risque de récidive en cas de non réintervention. Ainsi, une évaluation ganglionnaire systématique par la technique du ganglion sentinelle pour les patientes estimée à bas risque en préopératoire pourrait être une alternative intéressante.

Remerciements : Vincent Lavoué reçoit des subventions de la part de la Vannetaise (2014 et 2015) pour la recherche sur les cancers féminins. 


\section{Bibliographie}

1. Binder-Foucard F, Belot A, Delafosse P, Remontet L, Woronoff A-S, Bossard N. Estimation nationale de l'incidence et de la mortalité par cancer en France entre 1980 et 2012. Partie 1 - Tumeurs solides. Saint-Maur- ice (Fra): Institut de veille sanitaire; 2013, $122 \mathrm{p}$.

2. Creasman WT, Odicino F, Maisonneuve P, et al. Carcinoma of the corpus uteri. Int $\mathbf{J}$ Gynaecol Obstet 2003;83 Suppl 1:79-118.

3. Siegel R, Naishadham D, Jemal A. Cancer statistics, 2013. CA Cancer J Clin $2013 ; 63: 1130$.

4. Recommandations de l'INCa novembre 2010.http://www.ecancer.fr/soins/recommandations/cancer-gynécologiques.

5. S. Pecorelli, "Revised FIGO staging for carcinoma of the vulva, cervix, and endometrium," International Journal of Gynecology \& Obstetrics, vol. 105, no. 2, pp. 103-104, May 2009.

6. M. M. Baekelandt, M. Castiglione, and On behalf of the ESMO Guidelines Working Group, "Endometrial carcinoma: ESMO Clinical Recommendations for diagnosis, treatment and follow-up," Annals of Oncology, vol. 20, no. suppl 4, pp. iv29-iv31, May 2009.

7. G. Plataniotis, M. Castiglione, and On behalf of the ESMO Guidelines Working Group, "Endometrial cancer: ESMO Clinical Practice Guidelines for diagnosis, treatment and follow-up," Annals of Oncology, vol. 21, no. Supplement 5, pp. v41v45, May 2010.

8. N. Colombo, E. Preti, F. Landoni, S. Carinelli, A. Colombo, C. Marini, C. Sessa, and On behalf of the ESMO Guidelines Working Group, "Endometrial cancer: ESMO Clinical Practice Guidelines for diagnosis, treatment and follow-up," Annals of Oncology, vol. 22, no. Supplement 6, pp. vi35-vi39, Sep. 2011. 
9. N. Colombo, E. Preti, F. Landoni, S. Carinelli, A. Colombo, C. Marini, C. Sessa, and on behalf of the ESMO Guidelines Working Group, "Endometrial cancer: ESMO Clinical Practice Guidelines for diagnosis, treatment and follow-up," Annals of Oncology, vol. 24, no. suppl 6, pp. vi33-vi38, Oct. 2013.

10. W. M. Burke, J. Orr, M. Leitao, E. Salom, P. Gehrig, A. B. Olawaiye, M. Brewer, D. Boruta, J. Villella, T. Herzog, and F. Abu Shahin, "Endometrial cancer: A review and current management strategies: Part I," Gynecologic Oncology, vol. 134, no. 2, pp. 385-392, Aug. 2014.

11. W. M. Burke, J. Orr, M. Leitao, E. Salom, P. Gehrig, A. B. Olawaiye, M. Brewer, D. Boruta, T. J. Herzog, F. A. Shahin, and others, "Endometrial cancer: A review and current management strategies: Part II," Gynecologic oncology, vol. 134, no. 2, pp. 393-402, 2014.

12. Compte rendu normalisé réalisé par la Société française de radiologie (SFR).Source : site internet de la SFR. http://www.sfrnet.org/portal/site/societe/5-groupes-detravail/2-Transversaux/groupe-imagerie-cancérologique/comptes-rendusnormalises/index.phtml

13. H. C. Kitchener and E. L. Trimble, "Endometrial Cancer State of the Science Meeting:," International Journal of Gynecological Cancer, vol. 19, no. 1, pp. 134140, Jan. 2009.

14. S. Bendifallah, G. Canlorbe, P. Collinet, E. Arsene, F. Huguet, C. Coutant, D. Hudry, O. Graesslin, E. Raimond, C. Touboul, E. Darai, and M. Ballester, “Just how accurate are the major risk stratification systems for early-stage endometrial cancer?," $\mathrm{Br} J$ Cancer, vol. 112, no. 5, Mar. 2015.

15. E. Raimond, G. Canlorbe, S. Bendifallah, D. Hudry, F. Selvi, M. Ballester, C. Coutant, O. Graesslin, and E. Darai, "[Endometrial carcinoma. Application of the guidelines of 2010: multicentre trial].," Bull Cancer, vol. 101, no. 7-8, pp. 703-713, Aug. 2014. 
16. Frei KA, Kinkel K, Bonél HM, Lu Y, Zaloudek C, Hricak H. Prediction of deep myometrial invasion in patients with endometrial cancer: clinical utility of contrastenhanced MR. Radiology 2000;216:444-9

17. E. Sala, A. Rockall, and R. A. Kubik-Huch, "Advances in magnetic resonance imaging of endometrial cancer.," Eur Radiol, vol. 21, no. 3, pp. 468-473, Mar. 2011

18. Faria SC, Sagebiel T, Balachandran A, Devine C, Lal C, Bhosale PR. Imaging in endometrial carcinoma. The Indian Journal of Radiology \& Imaging. 2015;25(2):137147. doi:10.4103/0971-3026.155857

19. Frumovitz M, Singh DK, Meyer L, et al. Predictors of final histology in patients with endometrial cancer. Gynecol Oncol 2004;95:463-8.

20. Huang GS, Gebb JS, Einstein MH, Shahabi S, Novetsky AP, Goldberg GL. Accuracy of preoperative endometrial sampling for the detection of high-grade endometrial tumors. Am J Obstet Gynecol 2007;196:243 e1-5.

21. Groff B, Pouget O, Stoll F, Mathelin C, Baldauf JJ, Akladios CY. [Pertinence of the preoperative exploration in the evaluation of the risk of lymph node metastasis in endometrial cancer]. Gynecol Obstet Fertil 2014;42:92-6.

22. S. Gilani, I. Anderson, L. Fathallah, and P. Mazzara, "Factors predicting nodal metastasis in endometrial cancer," Archives of Gynecology and Obstetrics, vol. 290, no. 6, pp. 1187-1193, Dec. 2014.

23. M. Koskas, K. Bassot, O. Graesslin, P. Aristizabal, E. Barranger, F. Clavel-Chapelon, B. Haddad, D. Luton, E. Darai, and R. Rouzier, "Impact of lymphovascular space invasion on a nomogram for predicting lymph node metastasis in endometrial cancer," Gynecologic Oncology, vol. 129, no. 2, pp. 292-297, May 2013.

24. S. Bendifallah, G. Canlorbe, E. Arsène, P. Collinet, F. Huguet, C. Coutant, D. Hudry, O. Graesslin, E. Raimond, C. Touboul, E. Daraï, and M. Ballester, "French Multicenter Study Evaluating the Risk of Lymph Node Metastases in Early-Stage Endometrial Cancer: Contribution of a Risk Scoring System,” Annals of Surgical Oncology, Jan. 2015. 
25. S. R. Guntupalli, I. Zighelboim, N. T. Kizer, Q. Zhang, M. A. Powell, P. H. Thaker, P. J. Goodfellow, and D. G. Mutch, "Lymphovascular space invasion is an independent risk factor for nodal disease and poor outcomes in endometrioid endometrial cancer.," Gynecol Oncol, vol. 124, no. 1, pp. 31-35, Jan. 2012.

26. M. M. AlHilli, K. C. Podratz, S. C. Dowdy, J. N. Bakkum-Gamez, A. L. Weaver, M. E. McGree, G. L. Keeney, W. A. Cliby, and A. Mariani, "Risk-scoring system for the individualized prediction of lymphatic dissemination in patients with endometrioid endometrial cancer.," Gynecol Oncol, vol. 131, no. 1, pp. 103-108, Oct. 2013.

27. P. Aristizabal, O. Graesslin, E. Barranger, F. Clavel-Chapelon, B. Haddad, D. Luton, E. Darai, R. Rouzier, and M. Koskas, "A suggested modification to FIGO stage I endometrial cancer,” Gynecologic Oncology, vol. 133, no. 2, pp. 192-196, May 2014.

28. Hirschowitz L, Nucci M, Zaino RJ. Problematic issues in the staging of endometrial, cervical and vulval carcinomas. Histopathology 2013;62:176-202.

29. N. Agar, A.-C. Philippe, N. Bourdel, B. Rabischong, M. Canis, G. Le Bouedec, A. Mulliez, J. Dauplat, and C. Pomel, "Les lymphadénectomies dans le cancer de l'endomètre, bilan après 4ans de pratique, doit-on poursuivre?," Bulletin du Cancer, May 2015.

30. P. Benedetti Panici, S. Basile, M. G. Salerno, V. Di Donato, C. Marchetti, G. Perniola, A. Palagiano, A. Perutelli, F. Maneschi, A. A. Lissoni, M. Signorelli, G. Scambia, S. Tateo, G. Mangili, D. Katsaros, E. Campagnutta, N. Donadello, S. Greggi, M. Melpignano, F. Raspagliesi, G. Cormio, R. Grassi, M. Franchi, D. Giannarelli, R. Fossati, V. Torri, C. Croce, and C. Mangioni, "Secondary analyses from a randomized clinical trial: age as the key prognostic factor in endometrial carcinoma.," Am J Obstet Gynecol, vol. 210, no. 4, Apr. 2014.

31. M. Morneau, W. Foster, M. Lalancette, T. Van Nguyen-Huynh, M.-C. Renaud, V. Samouëlian, and N. Letarte, “Adjuvant treatment for endometrial cancer: Literature review and recommendations by the Comité de l'évolution des pratiques en oncologie (CEPO)," Gynecologic Oncology, vol. 131, no. 1, pp. 231-240, Oct. 2013. 
32. Kitchener H, Swart AM, Qian Q, Amos C, Parmar MK. Efficacy of systematic pelvic lymphadenectomy in endometrial cancer (MRC ASTEC trial): a random- ised study. Lancet 2009;373(9658):125-36

33. Benedetti Panici P, Basile S, Maneschi F, Alberto Lissoni A, Signorelli M, Scambia G, et al. Systematic pelvic lymphadenectomy vs no lymphadenecto- my in early-stage endometrial carcinoma: randomized clinical trial. $\mathrm{J}$ Natl Cancer Inst 2008;100(23):1707-16.

34. JA Frost, KE Webster, A Bryant, J Morrison. Lymphadenectomy for the management of endometrial cancer. Cochrane Database Syst Rev 2015 Sep 21 ; :CD007585

35. M. Ballester, G. Dubernard, F. Lécuru, D. Heitz, P. Mathevet, H. Marret, D. Querleu, F. Golfier, E. Leblanc, R. Rouzier, and others, "Detection rate and diagnostic accuracy of sentinel-node biopsy in early stage endometrial cancer: a prospective multicentre study (SENTI-ENDO)," The lancet oncology, vol. 12, no. 5, pp. 469-476, 2011. 
Tableau 1. Caractéristiques de la population

\section{Table 1: Characteritics of patients}

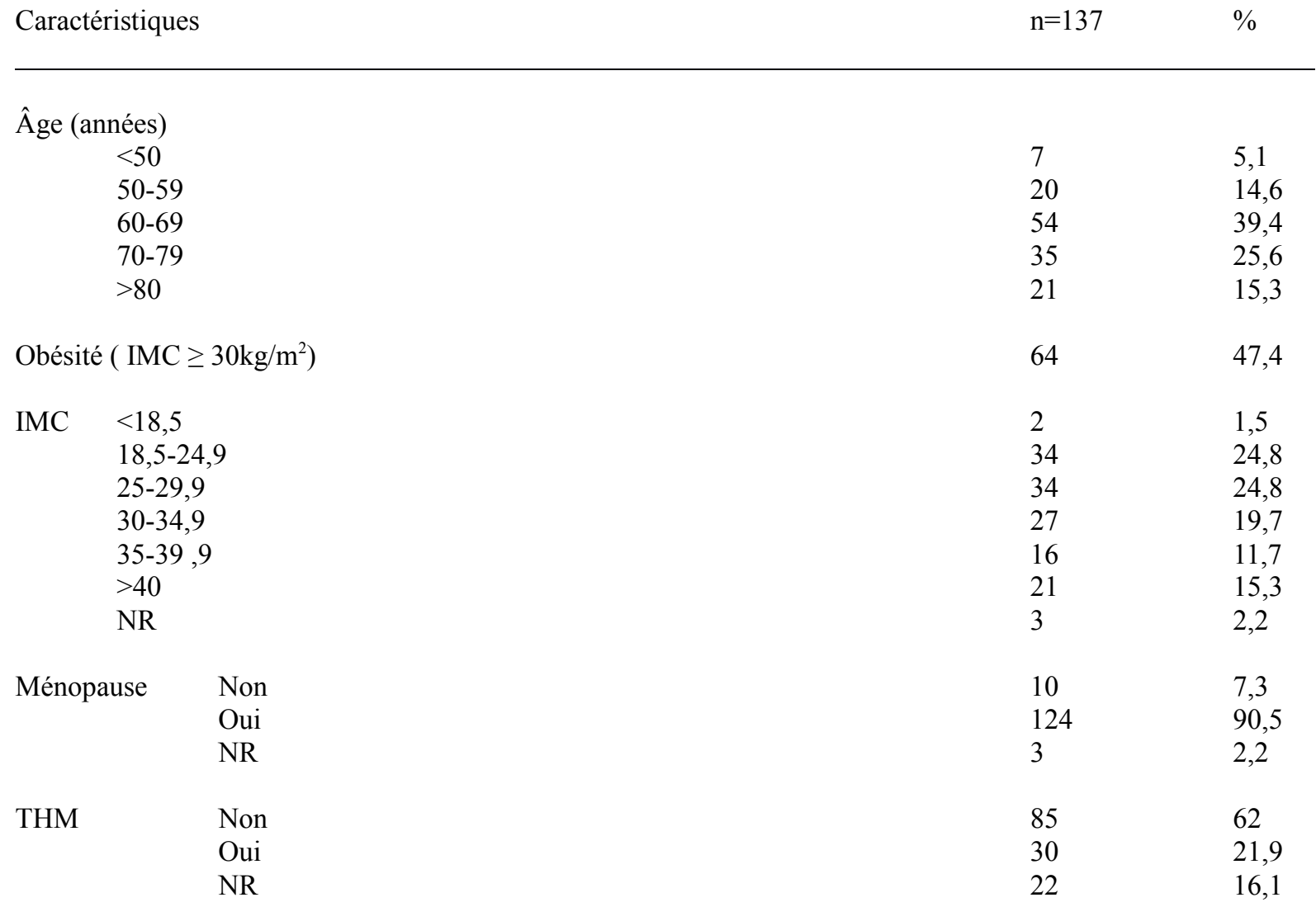

IMC : indice de masse corporelle ; THM : traitement hormonal de la ménopause ; NR : non renseigné 
Tableau 2. Bilan préopératoire

Table 2 : pre-operative data

Caractéristiques

$\mathrm{n}=137$

$\%$

$\begin{array}{ll}\text { Type de prélèvement } & \begin{array}{l}\text { Pipelle Cormier } \\ \text { Hystéroscopie curetage } \\ \text { Autres }\end{array} \\ \text { Type histologique } & \begin{array}{l}\text { Tumeurs endométrioïdes (type1) } \\ \text { Carcinomes à c.claires, papillaires séreux et carcinosarcomes (type 2) } \\ \text { NR }\end{array}\end{array}$

21,5

$\begin{array}{ll}\text { Tumeurs endométrioïdes } & \text { Grade 1 } \\ & \text { Grade } 2 \\ & \text { Grade } 3 \\ & \text { NR } \\ & \\ \text { Imagerie préopératoire } & \text { IRM } \\ & \text { IRM avec CR complet }{ }^{*} \\ & \text { TDM } \\ & \text { Echographie } \\ & \text { TEP } \\ & \text { Pas d'imagerie préopératoire }\end{array}$

$\begin{array}{ll}50 & 43,8 \\ 49 & 43,0 \\ 6 & 5,3 \\ 9 & 7,9\end{array}$

Envahissement myométrial $\quad<50 \%$ préopératoire $>\mathrm{Ou}=50 \%$

$\begin{array}{llll}\text { Stade FIGO préopératoire } & \text { IA } & 55 & 25 \\ & \text { IB } & 40,1 & 18,2 \\ & \text { II } & 1,5 \\ & \text { III } & 14,0 & 2 \\ & \text { IV } & 19 & 1 \\ & \text { NR } & 35 & 25,7\end{array}$

Classification de risque d'envahissement ganglionnaire selon l'INCa

\author{
Risque faible \\ Risque intermédiaire \\ Risque élevé***
}

Risque indéterminable ${ }^{* * *}$

* CR complet : Compte rendu complet défini par l'information de l'envahissement myométrial et la précision de l'existence d'adénopathies pelviennes et lomboaortiques

** Stade IB grade 3, stade IA et IB type II, stade II, III et IV type I et II

*** Bilan préopératoire incomplet ou avec compte rendu incomplet 
Tableau 3. Stadification chirurgicale réalisée en fonction de la classification du risque pré et post-opératoire d'envahissement ganglionnaire

\section{Table 3 : Surgery according lymph node involvment risk classification}

\begin{tabular}{|c|c|c|}
\hline Risque défini selon bilan préopératoire & $\mathrm{n}=137$ & $\%$ \\
\hline \multicolumn{3}{|l|}{ Stadification chirurgicale } \\
\hline Recommandée & 70 & $51,1 \%$ \\
\hline \multirow[t]{3}{*}{ Non recommandée } & 32 & $23,4 \%$ \\
\hline & 20 & $14,6 \%$ \\
\hline & 12 & $8,8 \%$ \\
\hline Risque préopératoire non déterminé & 35 & $25,5 \%$ \\
\hline Risque défini selon histologie postopératoire & $\mathrm{n}=137$ & $\%$ \\
\hline \multicolumn{3}{|l|}{ Prise en charge chirurgicale } \\
\hline Recommandée & 101 & $73,7 \%$ \\
\hline \multirow{2}{*}{ Non recommandée } & 36 & $26,3 \%$ \\
\hline & 36 & $26,3 \%$ \\
\hline
\end{tabular}

* Réalisation d'un curage pelvien et/ou lomboaortique non recommandé

** Absence de réalisation d'un curage pelvien et/ou lomboaortique recommandé 
Tableau 4. Histologie définitive sur pièce opératoire Table 4 : Pathological data

\begin{tabular}{|c|c|c|}
\hline \multicolumn{3}{|l|}{ Type Histologique } \\
\hline $\begin{array}{l}\text { Post-opératoire } \\
\text { Pré-opératoire }\end{array}$ & $\mathrm{I}$ & II \\
\hline $\mathrm{I}(\mathrm{n}=114) 83,2 \%$ & 107 & 7 \\
\hline II $(n=17) 12,4 \%$ & 6 & 11 \\
\hline $\operatorname{NR}(n=6) 4,4 \%$ & 5 & 1 \\
\hline & $118(86,1 \%)$ & $19(13,9 \%)$ \\
\hline
\end{tabular}

\begin{tabular}{|c|c|c|c|c|}
\hline \multicolumn{5}{|l|}{ Grade histopronostique } \\
\hline Postopératoire & 1 & 2 & 3 & NR \\
\hline \multicolumn{5}{|l|}{ Préopératoire } \\
\hline $1(n=50) 36,5 \%$ & 42 & 5 & 2 & 1 \\
\hline $2(n=49) 35,8 \%$ & 18 & 21 & 9 & 1 \\
\hline $3(n=13) 9,5 \%$ & 0 & 2 & 11 & 0 \\
\hline \multirow[t]{2}{*}{ NR $(n=25) 18,2 \%$} & 8 & 7 & 8 & 2 \\
\hline & $68(49,6 \%)$ & $35(25,6 \%)$ & $30(21,9 \%)$ & $4(2,9 \%)$ \\
\hline
\end{tabular}

\begin{tabular}{|l|llc|}
\hline \multicolumn{1}{|c|}{$\begin{array}{c}\text { Histologie } \\
\text { postopératoire }\end{array}$} & Bas & Intermédiaire & Elevé \\
Bilan préopératoire & & & \\
\hline Bas (45) & $27(60 \%)$ & $7(15,6 \%)$ & $11(24,4 \%)$ \\
\hline Intermédiaire (24) & $4(16,7 \%)$ & $7(29,1 \%)$ & $13(54,2 \%)$ \\
\hline Elevé (33) & $3(9,1 \%)$ & $2(6,0 \%)$ & $28(84,9 \%)$ \\
\hline Indéterminé $(35)$ & $15(42,9 \%)$ & $6(17,1 \%)$ & $14(40,0 \%)$ \\
\hline
\end{tabular}

